\title{
A systematic review of the use of subcortical intraoperative electrical stimulation mapping for monitoring of executive deficits and neglect: what is the evidence so far?
}

\author{
Maud J. F. Landers ${ }^{1,2}$ (D) Margriet M. Sitskoorn ${ }^{2} \cdot$ Geert-Jan M. Rutten $^{1} \cdot$ Emmanuel Mandonnet $^{3,4,5}$. $^{2}$ \\ Wouter De Baene ${ }^{2}$
}

Received: 3 May 2021 / Accepted: 21 August 2021 / Published online: 21 October 2021

(c) The Author(s) 2021

\begin{abstract}
Background Over the past decade, the functional importance of white matter pathways has been increasingly acknowledged in neurosurgical planning. A method to directly study anatomo-functional correlations is direct electrical stimulation (DES). DES has been widely accepted by neurosurgeons as a reliable tool to minimize the occurrence of permanent postoperative motor, vision, and language deficits. In recent years, DES has also been used for stimulation mapping of other cognitive functions, such as executive functions and visuospatial awareness.

Methods The aim of this review is to summarize the evidence so far from DES studies on subcortical pathways that are involved in visuospatial awareness and in the following three executive functions: (1) inhibitory control, (2) working memory, and (3) cognitive flexibility.

Results Eleven articles reported on intraoperative electrical stimulation of white matter pathways to map the cognitive functions and explicitly clarified which subcortical tract was stimulated. The results indicate that the right SLF-II is involved in visuospatial awareness, the left SLF-III and possibly the right SLF-I are involved in working memory, and the cingulum is involved in cognitive flexibility.

Conclusions We were unable to draw any more specific conclusions, nor unequivocally establish the critical involvement of pathways in executive functions or visuospatial awareness due to the heterogeneity of the study types and methods, and the limited number of studies that assessed these relationships. Possible approaches for future research to obtain converging and more definite evidence for the involvement of pathways in specific cognitive functions are discussed.
\end{abstract}

Keywords Brain mapping $\cdot$ Direct electrical stimulation $\cdot$ Executive functions $\cdot$ Subcortical pathways $\cdot$ Tractography

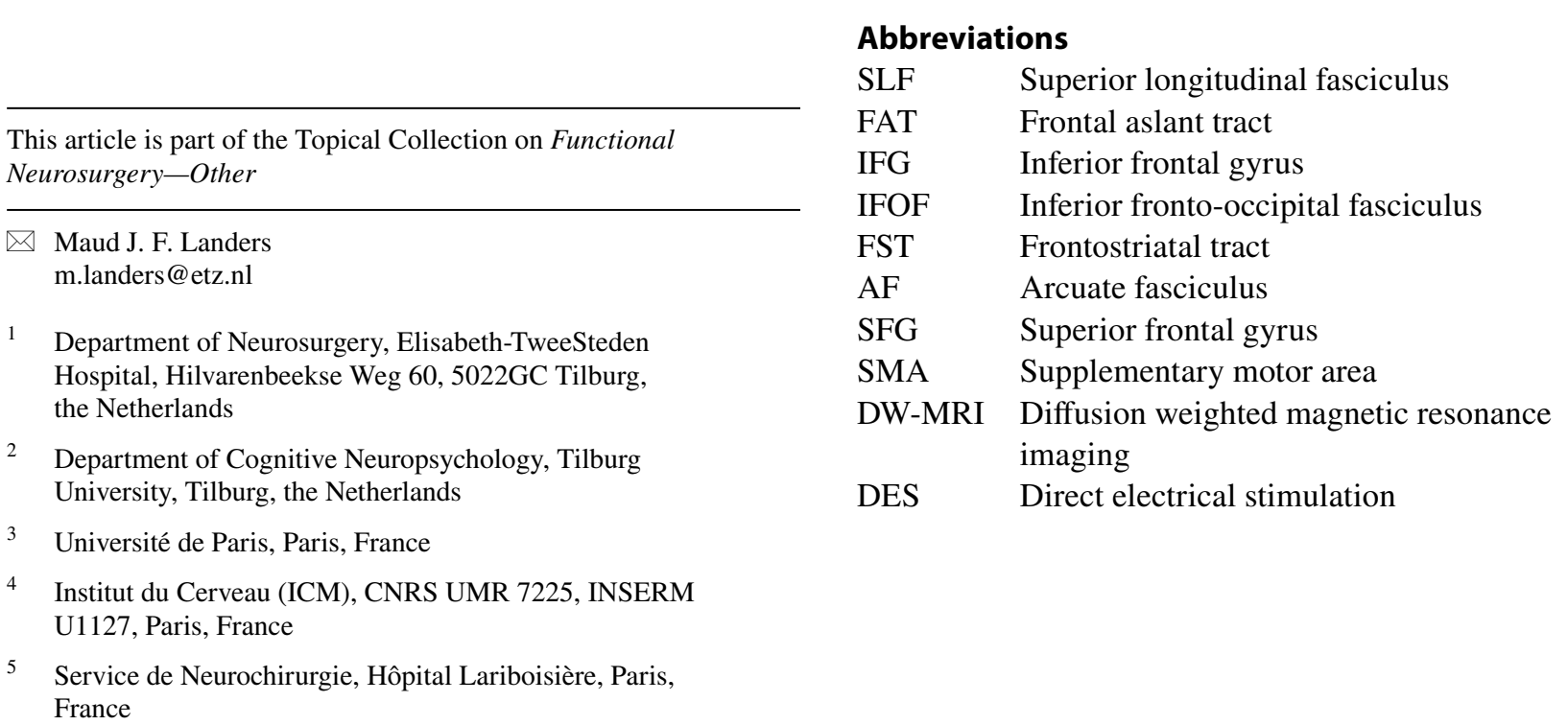




\section{Introduction}

Over the past decade, the functional importance of white matter pathways has been increasingly acknowledged in neurosurgical planning. Novel subcortical pathways have been identified by new neuroimaging and tractography techniques [38, 43]. Additional information on the functionality and clinical relevance of these pathways has come from lesion-symptom and brain mapping studies [8, 24, 84].Together, these anatomical and functional findings contributed to a shift from a predominantly localized view on functional topography towards a more network-based view. Specific brain functions are no longer solely attributed to a certain location on the cortex, but are thought to be represented by a particular network of cortical areas that are connected via subcortical pathways $[8,15,20$, $38,41,43,73]$.

A method to directly study anatomo-functional correlations is direct electrical stimulation (DES). DES has been widely accepted by neurosurgeons as a reliable tool for the identification of functional areas during surgery. It is currently considered the clinical gold standard for this purpose [50]. Historically, DES has been predominantly used to identify motor and language cortical areas [25, $30,32,49,72]$. In the past decades, it has also been routinely used to map the subcortical anatomy [9], and, more recently, to map cognitive functions other than language, such as executive functions and visuospatial awareness [23]. The rationale for intraoperative mapping of these latter functions is that cognitive and visuospatial impairments occur relative frequently among patients with glial tumours, both before and after surgery [69]. Several studies demonstrated that executive function impairments three months after surgery in glioma patients are frequent $[14,61]$. More specifically, a recent study showed that long-lasting executive function impairments in low-grade glioma patients are related to surgical disconnections of frontal and parietal white matter pathways [17]. Patients with executive functions impairment exhibit for example difficulties with planning, initiation, regulation and verification of complex, goal-directed behaviour [40] which negatively impact normal daily, social and professional life $[21,26,55]$ and health-related quality of life [1]. Patients with visuospatial deficits also exhibit a diminished postoperative quality of life [22, 39, 60].

The nomenclature of executive functions is widely discussed in the literature, but these functions can generally be described as 'a set of general-purpose control mechanisms that regulate the dynamics of human cognition and action' [53]. There is a general agreement that the following three mutually correlated, but distinct core executive functions can be distinguished: (1) inhibitory control, (2) working memory, and (3) cognitive flexibility [21, 28, 53, 54]. (1) The first core executive function, inhibitory control, is the ability to control oneself and the ability to control attention. Both are endogenous, top-down processes necessary for active, goal-driven execution of cognitive functions $[34,65,85]$. Self-control involves the inhibition of impulses, old habits, thoughts of action and external stimuli, and allows one to change and choose our reactions. Deficits in inhibitory control of oneself may for example become manifest as the so-called frontal syndrome (i.e., deprecated term used for psychopathological conditions after frontal lesions) [2, 83]. Inhibitory control of attention is required for selective attention and focus, and to suppress or ignore interfering stimuli based on one's intentions or goals. Deficits in inhibitory control of attention may impair the distribution of directed attention, and may lead to concentration difficulties and sensory overload [85]. (2) The second core executive function, working memory, is generally described as a temporary storage system in which information can be processed and manipulated in a goal-driven context [3]. There are two types of working memory: verbal working memory, necessary for spoken and written language, and visuospatial working memory, necessary for recognition and processing of visual and spatial information [80]. Deficits in working memory may result in anterograde amnesia and lead to problems with, for example reasoning, decisionmaking and behaviour in general $[5,21]$. Working memory and inhibitory control generally co-occur, as working memory is needed to remember what is relevant and what should be inhibited (e.g., concentrating on a goal). Vice versa, inhibitory control is necessary for selection and priority setting (e.g., ignoring distractions), which are needed to direct information for temporal storage in working memory $[21,80]$. (3) The third core executive function, cognitive flexibility, is the ability to change perspective (i.e., to shift attention) by adapting to a new set of norms, demands or priorities. This function is needed to switch between tasks. Deficits in cognitive flexibility are related to a variety of attentional problems, for example difficulties to concentrate and oversensitivity $[88,90]$. These deficits are often reported in brain tumour patients, and more generally, in patients with brain damage [29, 31]. Cognitive flexibility requires inhibition to deactivate the prior perspective and activation of the new perspective in working memory, demonstrating the commonality of the three executive functions [21].

Visuospatial awareness refers to the ability of identifying, processing and interpreting visual information about objects in space. Multimodel cognitive deficits due to lesions in several different networks (e.g., motor control network, ventral attention network) may lead to a reduction or loss of spatial awareness for the contralateral space, 
clinically resulting in 'neglect' $[7,11]$. It has been hypothesized that in hemispatial neglect a one-sided attentional bias occurs due to inhibition of the contralateral hemisphere. Note that the underlying spatial, inhibitory and attentional networks of neglect have not yet been fully elucidated [6, 36, 57, 87]. However, neglect often co-occurs with executive functional deficits and it is hypothesized that neglect may occur as a consequence of lesions to several regions involved in different executive networks [39]. Further evidence for this is found in rehabilitation studies, which demonstrate that rehabilitation programs that focus on spatial attention have not been able to alter most of the perceptual biases present in neglect, whereas rehabilitation strategies that focus also on spatial working memory capacity and executive control have been able to alter the perceptual biases present in neglect [81].

Several groups have started to use tasks to monitor executive deficits and neglect during surgery using DES [27, 67, $78,95]$. However, there is no consensus yet on intraoperative task protocols that are best suited for this purpose. This review summarizes the evidence so far from DES studies on subcortical pathways that are involved in executive functions and visuospatial awareness. This knowledge can guide neurosurgeons in their current practice, and is a first step towards a consensus protocol for cognitive monitoring.

\section{Methods}

\section{Literature search}

A systematic literature search was conducted to identify studies in which subcortical mapping during awake neurosurgical operations was used to investigate tracts involved in inhibition, working memory and cognitive flexibility. The databases Pubmed Central, Embase, Web of Science, and Cochrane Central were searched up to July 19, 2020. Search terms were established by the first author and were verified by the second and last author. Search strategies were built and performed by the first author (see Supplemental material 1). Inclusion criteria were studies investigating adult patients, with any type of brain tumour, who underwent awake surgery, in which direct electrical stimulation of subcortical pathways was performed, and by whom neuropsychological performance was measured. Eligible studies were clinical trials, randomized controlled trials, case series and case reports from all years of publication, written in English. Systematic reviews, narrative reviews and metaanalyses were also included initially. Letters, (conference) abstracts, and protocols were assessed as ineligible. Based on the results of our initial search we have added neglect to the search strategy.

\section{Study selection}

Studies were screened on title and abstract by the first and last author. Full text was screened if it was not clear from the abstract whether the study met the inclusion criteria. Screening results from both authors were compared and cases of doubt were discussed. Consensus was reached for all cases. Reviews and meta-analyses and reference lists from eligible articles were screened by the first author for additional articles.

\section{Assessment of executive functions and visuospatial awareness}

To ensure that all studies investigating executive functioning would be covered in the search strategy, a broader term and its derivatives were also added: neuropsychological performance, and cognition. In this way, we prevented studies assessing more than one cognitive function from being mistakenly excluded. Therefore, all abstracts were critically evaluated by the first and last author to examine whether inhibition, working memory, cognitive flexibility (or related terms), or visuospatial awareness were mentioned, even if this was not the main topic of the study. Note that studies examining higher cognitive functions that do not require executive control or visuospatial awareness were therefore not included in this review.

\section{Quality assessment of studies}

The quality of each of the included studies was assessed by the first and last author using the 8-item Methodological Index for Nonrandomized Studies (MINORS) for nonrandomized studies [79]. In case of a comparative study, 4 additional items were scored. MINORS includes the assessment of risk of bias in individual studies. Consensus was reached for all cases.

\section{Results}

\section{Study selection}

The initial systematic literature search identified 575 articles (see Fig. 1) [56]. After removing duplicates and screening titles and abstracts, 116 articles remained that met the inclusion criteria. Full-text articles were assessed for eligibility, resulting in the inclusion of 18 articles. Twenty-one reviews were used for cross-referencing but 
Fig. 1 PRISMA flow diagram [51] presenting the selection of studies for DES of subcortical pathways involved in executive functions

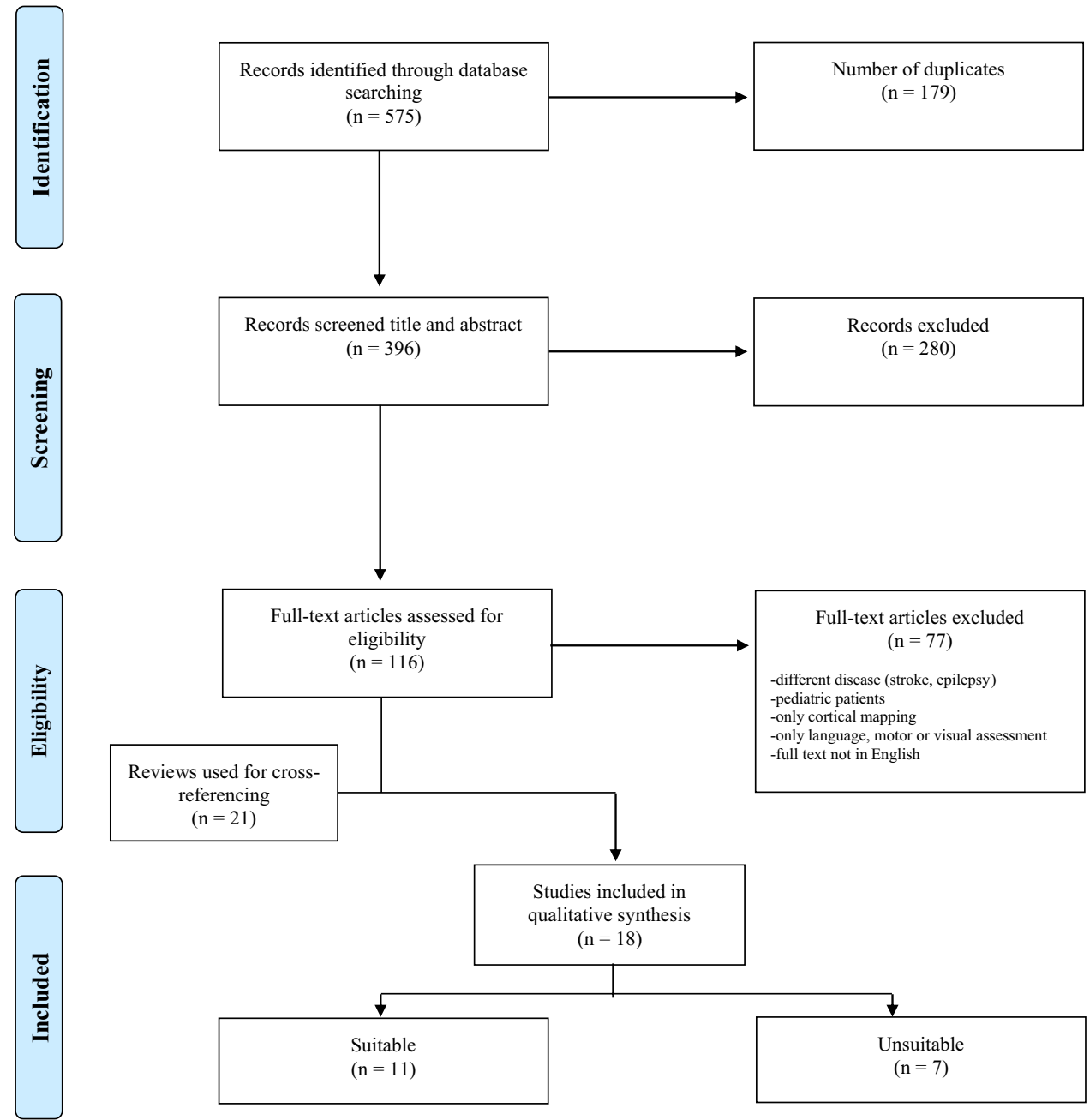

this did not lead to the inclusion of additional articles, nor did screening the reference lists of the eligible articles.

\section{Study characteristics}

Of the 18 eligible studies 11 were deemed to be suitable for this review because they provide a reliable estimate of the stimulated anatomical pathway, as well as the behavioural deficits during electrical stimulation mapping. The suitable included studies were conducted between 2005 and 2019, of which 4 were prospective mapping studies, 3 were case reports or case series, 3 were retrospective studies and 1 compared two cases with a control group. The results are presented in Table 1 and summarized below per function. The column 'Tract' includes all the tracts tested (positive and negative) for the functions. Two studies were deemed unsuitable because they assessed recognition [12, 51]. The other five studies that were deemed unsuitable did assess the functionality of subcortical pathways in executive functions or visuospatial awareness, but did not clearly indicate which subcortical pathway was stimulated and were therefore not considered suitable to answer the research questions of this review. All five unsuitable studies reported on inhibitory control and used the line bisection task $[18,59,74,75]$ or target cancellation task [18] to assess visuospatial awareness or an intraoperative Stroop test to assess inhibition of automatic responses ${ }^{44}$.

\section{Inhibitory control}

Only one of the eleven included studies reported on subcortical pathways involved in inhibitory control. This study investigated inhibitory control of oneself through performance on the Stroop task during subcortical electrical stimulation in 34 patients [66]. Their stimulation results indicate that the right inferior frontostriatal tract (FST) and the right thalamic radiation are involved in inhibitory control.

\section{Working memory}

Two of the eleven included studies reported on pathways involved in working memory. One study reported on 


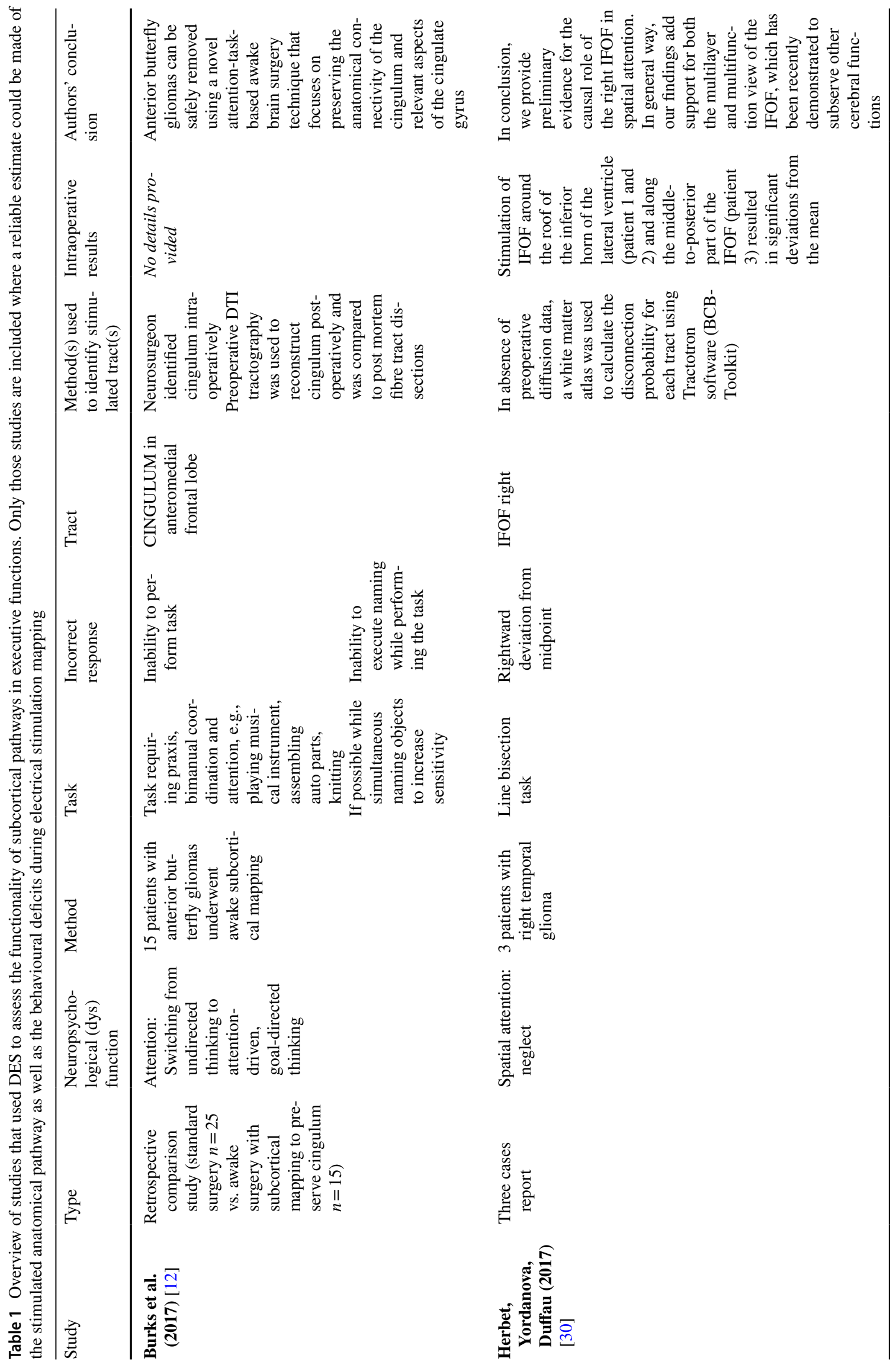




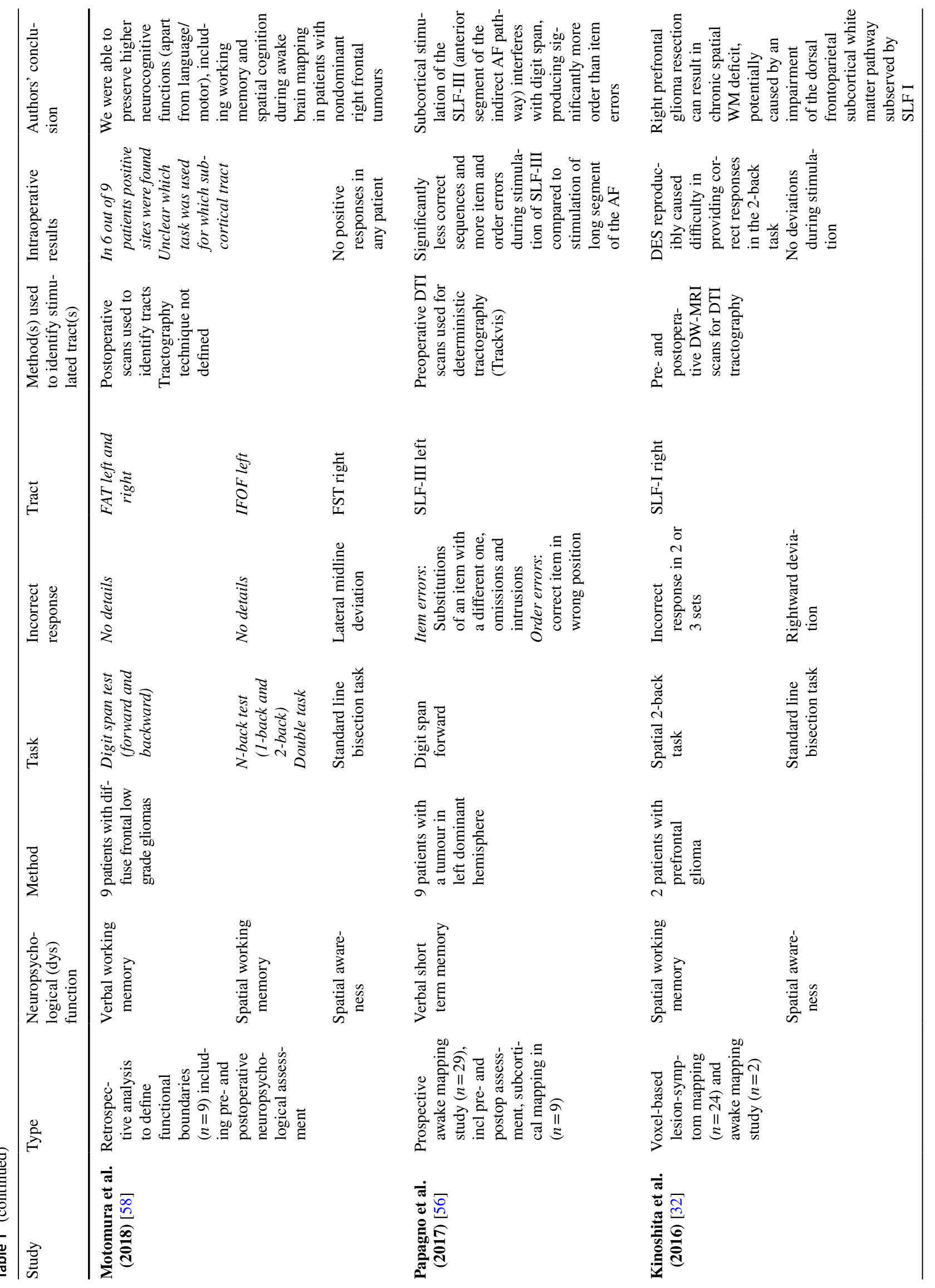




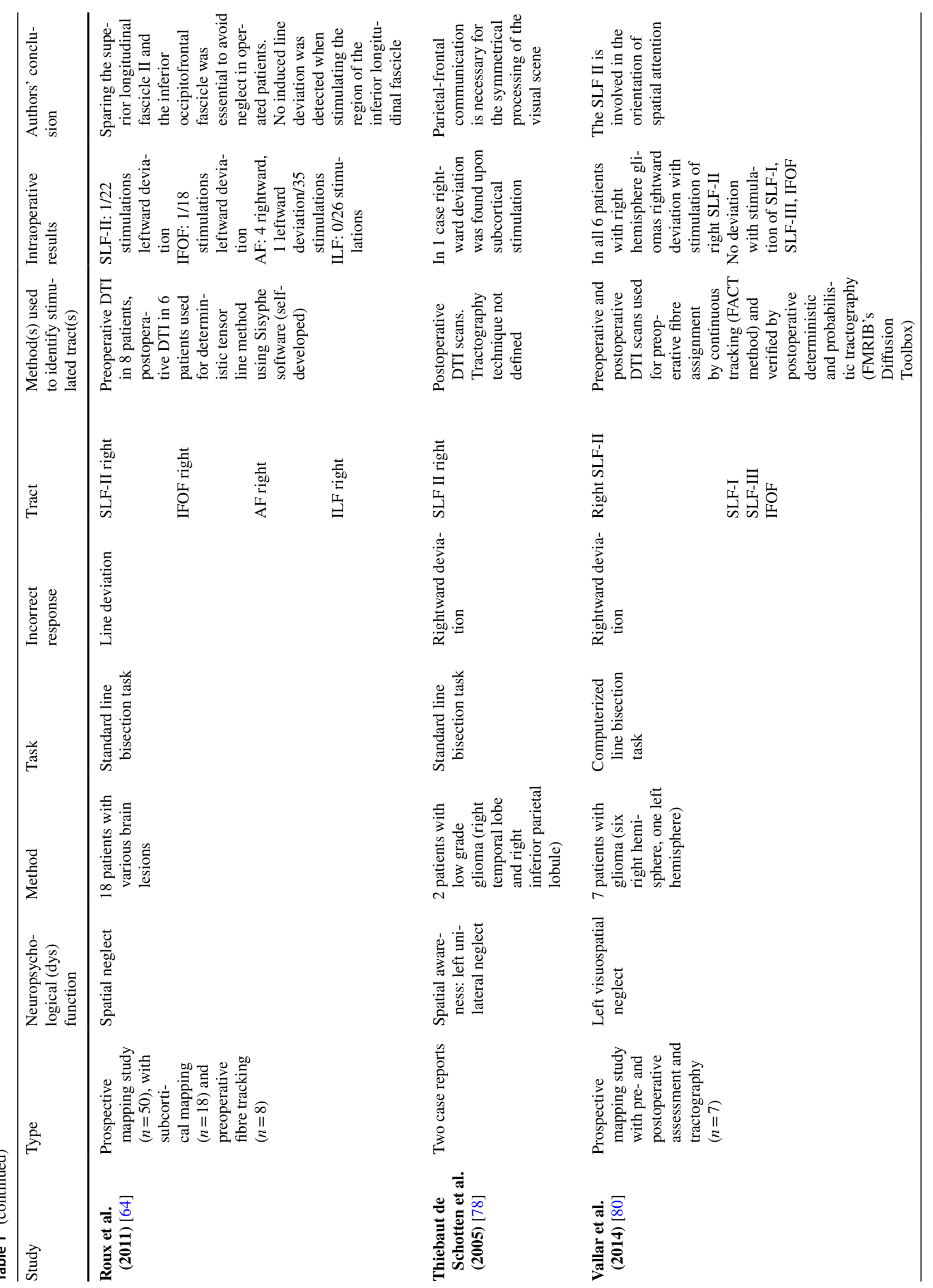




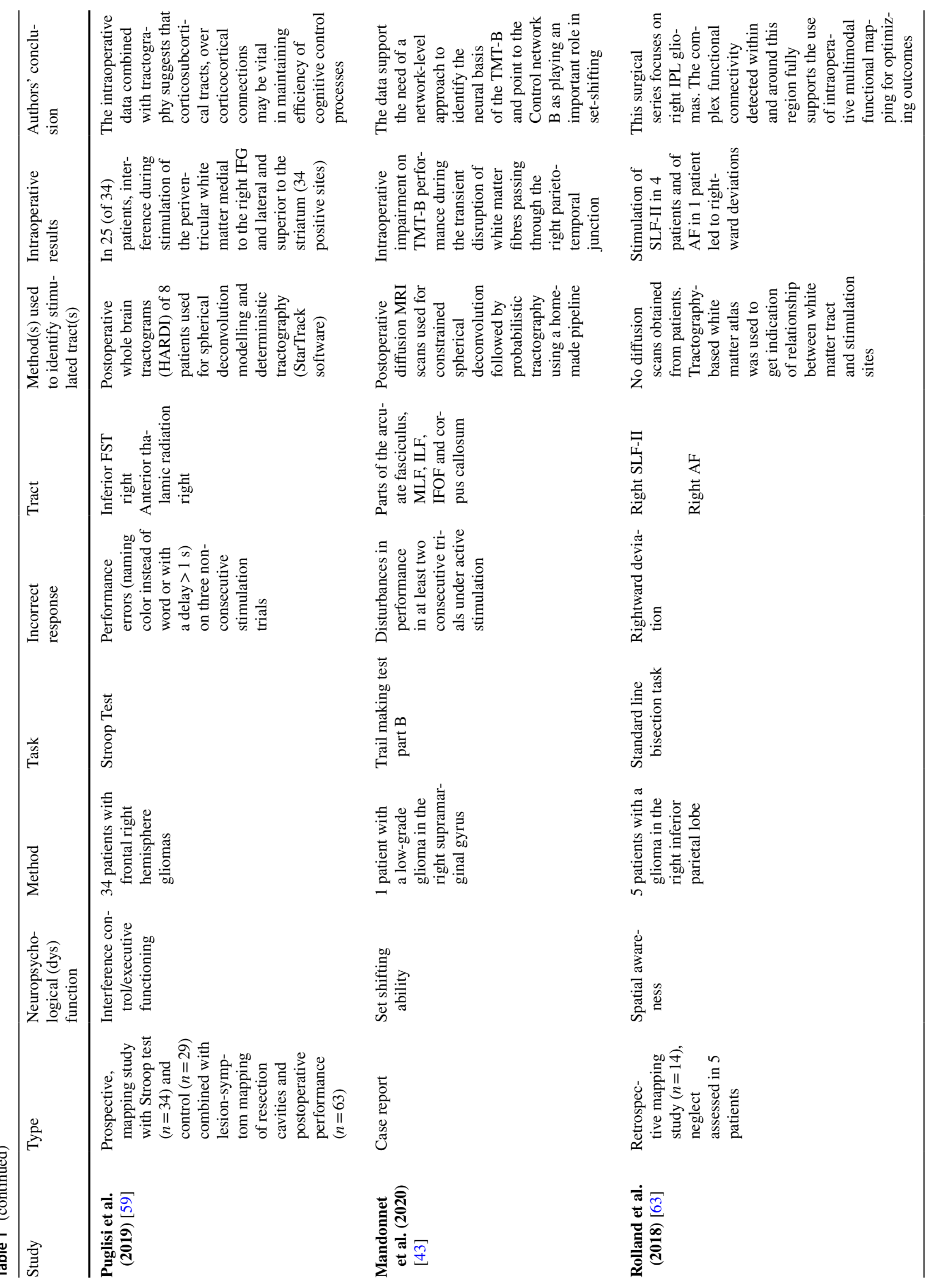


visuospatial working memory and showed that subcortical stimulation of the right SLF-I led to incorrect responses on a spatial N-back test in two patients. Note that this study also examined inhibitory control. The other study reported on verbal working memory and showed that subcortical stimulation of the left SLF-III in nine patients resulted in interferences with the digit span [63].

\section{Cognitive flexibility}

Two of the eleven included studies reported on pathway involvement in cognitive flexibility. One study concluded that stimulation of the cingulum leads to problems in the execution of a double task in 15 patients, although no intraoperative stimulation results were reported [13]. The other study was a case report in which set-shifting ability was investigated with the trail making test part B (TMT-B), showing that stimulation of white matter fibres passing through the right parieto-temporal junction leads to impaired performance on the TMT-B [48].

\section{Visuospatial awareness}

Seven of the included eleven studies investigated visuospatial awareness with a line bisection task and regarded deviation from the midpoint as an incorrect response indicating neglect. Incorrect responses were mainly found when stimulating the superior longitudinal fasciculus (SLF)-II. Across all studies that assessed right SLF-II, stimulation resulted in neglect in all 11 patients [70, 86, 89], suggesting that this pathway is involved in spatial attention. Negative responses (i.e., no intraoperative error on the task) were obtained from stimulations of the right SLF-I in 8 patients [35, 89], the right SLF-III in six patients [89] and the right FST (number of patients unknown) [58] suggesting that these tracts are not involved in spatial attention. Stimulation results for the right inferior fronto-occipital fasciculus (IFOF) and the right arcuate fasciculus (AF) are inconsistent and do not allow to make general conclusions. One study reported that electrical stimulation of the right IFOF resulted in neglect in 3 patients [33], whereas another study reported no neglect after stimulation of the right IFOF in six patients [89]. In another study the AF was only stimulated in one patient and resulted in neglect [70]. One study performed subcortical mapping of the right SLF-II, the right IFOF, the right AF and the right ILF in 18 cases [71], but only reported the number of positive and negative stimulations per pathway and not in how many patients they occurred, which complicates the interpretation of the results.

\section{Quality assessment}

The mean 8-item MINORS score for the eleven included studies varied between 6 and 12, mean 8.82 and standard deviation 1.75 (see Supplemental material 2). Three of the eleven studies were case-reports or case-series and could not be scored adequately on item 2, 7 and 8. Only four studies had an adequate control group, and the mean 12-item MINORS score for those comparative studies varied between 11 and 18, mean 15.60, and standard deviation 2.50. None of the studies reported on unbiased assessment of the study endpoint or on a prospective calculation of the study size.

\section{Discussion}

This systematic review aimed to determine the evidence so far from electrical stimulation studies on the involvement of subcortical pathways in executive functions and visuospatial awareness in patients with brain tumours. The results uncovered that the evidence from the few eligible studies was not sufficient to unequivocally establish the involvement of any subcortical pathway in higher cognitive functions. We found only eleven studies that explicitly clarified which subcortical tract was stimulated during electrical stimulation, and were, therefore, suitable for further analyses. Study characteristics, methods and tasks were heterogenous, and methodological quality varied. Clearly, neurosurgical groups are exploring new methods to assess cognitive functions during brain tumour surgery, but are far from consensus on protocols and indications. This complicated the comparison and summarization of findings. However, after reviewing the literature, the results indicate that the right SLF-II is involved in visuospatial awareness, the left SLF-III and possibly the right SLF-I are involved in working memory, and the cingulum is involved in cognitive flexibility. These (preliminary) findings do not only reflect the urge for more research on subcortical mapping of executive functions, but do also provide an important first step towards establishing the potential functional important pathways for neurosurgical practice.

\section{Inhibitory control}

We defined inhibitory control as the ability to control oneself and the ability to control one's attention, needed to suppress goal-irrelevant stimuli and responses. A commonly used test to assess resistance to interference is the Stroop Colour and Word test [82]. Although several studies used this test to identify areas involved in interference control during cortical mapping [67, 95], only one study investigated performance on the Stroop task during subcortical electrical stimulation 
and indicate that the right inferior FST and the right thalamic radiation are involved in interference control [66].

\section{Working memory}

We defined working memory as a temporary storage system, needed to process and manipulate verbal and visuospatial information in a goal-driven context. Our results suggest that the left SLF-III is involved in verbal working memory and that the right SLF-I is possibly involved in visuospatial working memory. However, the one study that claimed involvement for the left SLF-III in verbal working memory only used a forward digit span task [63], suggesting impairment of recall rather than of executive functions $[4,96]$. Evidence from fMRI-studies in traumatic brain injury patients and brain tumour patients suggests that working memory deficits are the result of dysfunction of networks [76, 77]. When performing a working memory task, one has to switch from a resting state (i.e., default mode network) to a taskrelated active state (i.e., central executive network). As the SLF connects frontal and parietal areas of and within both these networks in both hemispheres [35, 37, 63], the SLF may play a central role in both verbal and spatial working memory. The fact that the SLF has three branches that connect different areas and that the SLF has different functional specializations for each hemisphere might explain why different functions were found for this tract $[10,16,44]$.

\section{Cognitive flexibility}

In this review, cognitive flexibility is defined as the ability to change perspective by adapting to a new set of norms, demands or priorities, which is needed to switch between tasks. Only two studies have reported on cognitive flexibility and their results suggest involvement of the cingulum and white matter fibres that cross the right parieto-temporal junction. A possible reason why cognitive flexibility is not often studied during awake neurosurgical procedures is that tests that measure this function are not particularly suited for the intraoperative setting. Although different forms of task switching tests have been used to measure cognitive flexibility [34, 92], these tests all rely on an ongoing sequence of task repeats and task switches, in which prolonged reaction time or making the wrong switch indicate problems in cognitive flexibility. Given that stimulation typically only lasts for four to six seconds, the ongoing aspect of task cues and the fact that stimulation may interfere with any presented cue, makes it very difficult to use this type of paradigm to measure cognitive flexibility during awake neurosurgical procedures.

Some studies use the TMT-B as a measure of cognitive flexibility and suggest this test is feasible for intraoperative use $[45,64,68]$. However, the TMT-B might not always be sensitive enough as a measure of cognitive flexibility. Complete disruption of cognitive flexibility will result in a shifting error that can be measured with the TMT-B. However, reduced cognitive flexibility will result in an increased reaction time on shifting trials, that can only be adequately interpreted if assessed together with the TMT part A (TMTA). A recent review concluded that alternating task performance primarily correlates with the TMT-B to TMT-A ratio (time measures). However, measuring both these tasks during surgery and using the ratio as a measure for performance at any given site, seems very challenging from a practical point of view [93]. Furthermore, another recent study found that TMT-B performance is greatly influenced by processing speed, just like the performance on shifting attention tasks, which complicates intraoperative use, where processing speed is already affected by the setting [42]. Additionally, the timing of stimulation and the stimulation location may distort performance on the cognitive flexibility task only through its effect on processing speed.

\section{Visuospatial awareness}

Deficits in visuospatial awareness may result in a disorder called neglect, which is defined as a disturbance in the spatial distribution of directed attention [52]. This disorder is often seen as a consequence of right parietal brain lesions that cause neglect in the left hemispace [19]. Current knowledge from electrical mapping studies suggests that the right SLF-II and possibly the right IFOF are involved in visuospatial awareness, whereas the right SLF-I, the right SLF-III and the right FST are not. Remarkably, in all but two studies [70, 89], the authors who described positive findings drew rather firm conclusions, as they stated that the subcortical tracts in question are 'necessary for' or 'essential for avoiding' neglect, while results were not clearly described or were only found in a single patient.

\section{Identification of stimulated tracts}

An important point when claiming functionality of a given tract is to know exactly what tract was electrically stimulated. The studies included used different methods to determine the subcortical white matter tracts that were stimulated (see Table 1). The methods varied from identification based on the neurosurgeon's anatomical knowledge, to identification with the help of navigated tractography. A couple of studies combined preoperative and postoperatively acquired tractography to reconstruct intraoperative stimulation sites and their spatial relation with fibre pathways [71]. Others used additional lesion-symptom mapping or disconnection analyses (to identify overlap with a white matter tract) [13, $35,48,67]$. However, none of these methods is free of (significant) error. Tractographic results are variable due to a 
great variety in techniques, anatomical uncertainty (physiological differences, disease related differences, undiscovered pathways), and a lack of standardization per method, because results depend on parameters chosen by the user [91]. Furthermore, inaccuracies of neuronavigational systems and additional intra-operative brain-shift may lead to errors in the documented spatial locations of the mapping sites [62]. Consequently, the possibility of inaccurate findings, false negatives and positives, and irreproducibility of stimulation results complicates the determination of anatomo-functional correlations. It addresses the need for consensus on an intraoperative mapping protocol for both stimulation and documentation of responses.

\section{Limitations}

We chose to present and discuss the results per cognitive function. However, all cognitive functions have a certain overlap in function and neurobiological underpinning, which complicates the interpretation of subcortical stimulation results. This review uses data that are acquired during neurosurgical procedures, in a setting that is obviously very different from a regular neuropsychological testing environment and only allows for one single-moment assessment (and not for assessment of performance over time). The tasks used for the assessment of neuropsychological functions are not developed or validated for intraoperative use and normally lack ecological validity (i.e., translatability to daily life functioning). Moreover, there are few sites in the brain where one can selectively target a single pathway and these pathways are clearly part of (one of more) networks that as a whole perform specific functions. Hence, stimulation of a single tract (if possible) may result in disruption of distant networks, whilst the neuropsychological findings are attributed to the tested tract for understandable but incorrect reasons. Furthermore, for most long range associative tracts there is still a substantial variability in the nomenclature and taxonomy (e.g., AF and SLF [94]), which may again lead to an incorrect attribution of function to tract [47]. In addition, the stimulation responses also depend on the tissue that has already been resected. Although this is not necessarily a problem from a surgical point of view, it makes it difficult to assess the exact functionality of a tract. Furthermore, we noticed that most studies reported only the positive mapping sites (i.e., sites where errors were found during mapping) and not the so-called negative mapping sites. We believe that strict documentation of negative mappings is also necessary to validate hypotheses for specific white matter tracts. Finally, the methods and quality of the studies varied, and the different study types could not always be compared. Consequently, due to this heterogeneity that limited the comparison between studies, every study had to be placed in the correct perspective by the authors, and induced a potential risk of bias across studies.

\section{Future research}

When summarizing the findings from subcortical mapping studies and carefully considering their limitations, as well as the limitations of this study, it is evident that DES can provide us with unique and important information regarding the functionality of the subcortical pathways through the localization of transient functional deficits. It has the potential to move forward both clinical practice and neuroscience. However, the combined results from published DES studies are at the moment not sufficient to unequivocally determine the involvement of subcortical pathways in executive functions or visuospatial awareness. Only when cognitive tasks are carefully introduced into the operating room, DES will be able to function as a reliable tool to increase the safety of brain tumour resections. Recently, a roadmap was proposed that addresses the problem of data collection in DES studies and advocates a systematic analysis of evidence before using (novel) cognitive tasks intraoperatively [46]. This roadmap provides clear guidelines for future studies to obtain converging and more definite evidence, in which DES results should be combined with the results of lesion-deficit studies (to correlate between tract and function), functional imaging studies (to study the role of a tract in and between functional networks) and structural imaging studies (for accurate visualization and identification of tracts). Importantly, if we want to establish whether or not a certain pathway is indispensable for a particular function, and thus is relevant to neurosurgeons, postoperative (neuropsychological) assessment should be performed to assess the cognitive status of the patient and his or her quality of life in the long run. For future research we should work towards a uniform way of collecting DES findings of subcortical pathways. Only then, we can learn more about the intricate architecture of the brain substantiating executive functions and learn if their underlying pathways are indispensable for normal cognitive functioning.

\section{Conclusion}

The evidence regarding the use of electrical subcortical stimulation mapping for assessment of executive functions and visuospatial awareness is limited. This systematic review provides an indication for several subcortical pathways to be involved in different functions. More importantly, this review reflects the urge for the development of a standardized assessment protocol for intraoperative subcortical mapping of cognitive functions and can function as a stepping stone to reach consensus. This is important for neurosurgical 
practice to achieve optimal postoperative quality of life and for enhancement of knowledge in neuroscience.

Supplementary Information The online version contains supplementary material available at https://doi.org/10.1007/s00701-021-05012-w.

Author contribution Conception and study design (MJL, WDB, GJR), literature search and study selection (MJL, WDB), quality assessment (MJL, WDB), interpretation of results (MJL, WDB, GJR, MM, EM), writing first draft of manuscript (MJL), revising draft manuscript (MJL, WDB, GJR, EM, MM), and approval of final version to be published and agreement to be accountable for the integrity and accuracy of all aspects of the work (all authors).

Funding This study is funded by ZonMw, a Dutch national organization for Health Research and Development (project number: 10070012010006).

Data availability Upon request.

\section{Declarations}

Ethics approval Not applicable.

Conflict of interest The authors declare no competing interests.

Open Access This article is licensed under a Creative Commons Attribution 4.0 International License, which permits use, sharing, adaptation, distribution and reproduction in any medium or format, as long as you give appropriate credit to the original author(s) and the source, provide a link to the Creative Commons licence, and indicate if changes were made. The images or other third party material in this article are included in the article's Creative Commons licence, unless indicated otherwise in a credit line to the material. If material is not included in the article's Creative Commons licence and your intended use is not permitted by statutory regulation or exceeds the permitted use, you will need to obtain permission directly from the copyright holder. To view a copy of this licence, visit http://creativecommons.org/licenses/by/4.0/.

\section{References}

1. Aaronson NK, Taphoorn MJ, Heimans JJ, Postma TJ, Gundy CM, Beute GN, Slotman BJ, Klein M (2011) Compromised healthrelated quality of life in patients with low-grade glioma. J Clin Oncol 29:4430-4435

2. Alexander MP, Stuss DT Disorders of frontal lobe functioning. In: Seminars in neurology, 2000. vol 04. Copyright $\odot 2000$ by Thieme Medical Publishers, Inc., 333 Seventh Avenue, New ..., pp 427-438

3. Baddeley A (1992) Working memory. Science 255:556-559. https://doi.org/10.1126/science.1736359

4. Baddeley A (1996) Exploring the central executive. Q J Exp Psychol A 49:5-28

5. Baddeley A (2012) Working memory: theories, models, and controversies. Annu Rev Psychol 63:1-29

6. Bartolomeo P, Thiebaut de Schotten M, Chica AB (2012) Brain networks of visuospatial attention and their disruption in visual neglect. Front Hum Neurosci 6:110
7. Bartolomeo P, Thiebaut de Schotten M, Doricchi F (2007) Left unilateral neglect as a disconnection syndrome. Cereb Cortex 17:2479-2490

8. Bates E, Wilson SM, Saygin AP, Dick F, Sereno MI, Knight RT, Dronkers NF (2003) Voxel-based lesion-symptom mapping. Nat Neurosci 6:448-450

9. Berger MS, Kincaid J, Ojemann GA, Lettich E (1989) Brain mapping techniques to maximize resection, safety, and seizure control in children with brain tumors. Neurosurgery 25:786-792

10. Bernard F, Lemee JM, Ter Minassian A, Menei P (2018) Right hemisphere cognitive functions: from clinical and anatomic bases to brain mapping during awake craniotomy Part I: clinical and functional anatomy. World Neurosurg 118:348-359. https://doi. org/10.1016/j.wneu.2018.05.024

11. Bisiach E, Luzzatti C (1978) Unilateral neglect of representational space. Cortex 14:129-133

12. Brandling-Bennett EM, Bookheimer SY, Horsfall JL, Moftakhar P, Sedrak M, Barkulis CT, Gertsch JH, MacDougall MG, Boucharel W, Nuwer MR, Bergsneider M (2012) A paradigm for awake intraoperative memory mapping during forniceal stimulation. Neurocase 18:26-38. https://doi.org/10.1080/13554794. 2010.547509

13. Burks JD, Bonney PA, Conner AK, Glenn CA, Briggs RG, Battiste JD, McCoy T, O'Donoghue DL, Wu DH, Sughrue ME (2017) A method for safely resecting anterior butterfly gliomas: the surgical anatomy of the default mode network and the relevance of its preservation. J Neurosurg 126:1795-1811. https:// doi.org/10.3171/2016.5.Jns153006

14. Butterbrod E, Synhaeve N, Rutten G-J, Schwabe I, Gehring K, Sitskoorn M (2020) Cognitive impairment three months after surgery is an independent predictor of survival time in glioblastoma patients. J Neurooncol 149:103-111

15. Catani M (2007) From hodology to function. Brain 130:602-605

16. Catani M, de Schotten MT (2012) Atlas of human brain connections. Oxford University Press

17. Cochereau J, Lemaitre AL, Wager M, Moritz-Gasser S, Duffau H, Herbet G (2020) Network-behavior mapping of lasting executive impairments after low-grade glioma surgery. Brain Struct Funct. https://doi.org/10.1007/s00429-020-02131-5

18. Conner AK, Glenn C, Burks JD, McCoy T, Bonney PA, Chema AA, Case JL, Brunner S, Baker C, Sughrue M (2016) The use of the target cancellation task to identify eloquent visuospatial regions in awake craniotomies. Cureus 8:11

19. Corbetta M, Shulman GL (2011) Spatial neglect and attention networks. Annu Rev Neurosci 34:569-599

20. De Benedictis A, Duffau H (2011) Brain hodotopy: from esoteric concept to practical surgical applications. Neurosurgery 68:1709-1723. https://doi.org/10.1227/NEU.0b013e3182 124690

21. Diamond A (2013) Executive functions. Annu Rev Psychol 64:135-168

22. Drewes C, Sagberg LM, Jakola AS, Solheim O (2016) Quality of life in patients with intracranial tumors: does tumor laterality matter? J Neurosurg 125:1400-1407

23. Duffau H (2016) Stimulation mapping of myelinated tracts in awake patients. Brain Plasticity 2:99-113

24. Duffau H (2019) Surgical mapping. J Neurol Sci 405:52. https:// doi.org/10.1016/j.jns.2019.10.145

25. Duffau H, Capelle L, Sichez N, Denvil D, Lopes M, Sichez JP, Bitar A, Fohanno D (2002) Intraoperative mapping of the subcortical language pathways using direct stimulations. An anatomo-functional study. Brain 125:199-214. https://doi.org/ 10.1093/brain/awf016

26. Duffau H, Mandonnet E (2013) The "onco-functional balance" in surgery for diffuse low-grade glioma: integrating the extent of 
resection with quality of life. Acta Neurochir (Wien) 155:951957. https://doi.org/10.1007/s00701-013-1653-9

27. Erez Y, Assem M, Coelho P, Romero-Garcia R, Owen M, McDonald A, Woodberry E, Morris RC, Price SJ, Suckling J (2020) Intraoperative mapping of executive function using electrocorticography for patients with low-grade gliomas. Acta Neurochirurgica:1-11

28. Friedman NP, Miyake A, Robinson JL, Hewitt JK (2011) Developmental trajectories in toddlers' self-restraint predict individual differences in executive functions 14 years later: a behavioral genetic analysis. Dev Psychol 47:1410

29. Gehring K, Aaronson NK, Taphoorn MJ, Sitskoorn MM (2010) Interventions for cognitive deficits in patients with a brain tumor: an update. Expert Rev Anticancer Ther 10:1779-1795

30. Gogos AJ, Young JS, Morshed RA, Hervey-Jumper SL, Berger MS (2020) Awake glioma surgery: technical evolution and nuances. J Neurooncol 147:515-524

31. Gu L, Li J, Feng D-F, Cheng E-T, Li D-C, Yang X-Q, Wang B-C (2013) Detection of white matter lesions in the acute stage of diffuse axonal injury predicts long-term cognitive impairments: a clinical diffusion tensor imaging study. J Trauma Acute Care Surg 74:242-247

32. Henderson F, Abdullah KG, Verma R, Brem S (2020) Tractography and the connectome in neurosurgical treatment of gliomas: the premise, the progress, and the potential. Neurosurg Focus 48:E6. https://doi.org/10.3171/2019.11.Focus19785

33. Herbet G, Yordanova YN, Duffau H (2017) Left spatial neglect evoked by electrostimulation of the right inferior fronto-occipital fasciculus. Brain Topogr 30:747-756. https://doi.org/10.1007/ s10548-017-0574-y

34. Kiesel A, Steinhauser M, Wendt M, Falkenstein M, Jost K, Philipp AM, Koch I (2010) Control and interference in task switching-a review. Psychol Bull 136:849

35. Kinoshita M, Nakajima R, Shinohara H, Miyashita K, Tanaka S, Okita H, Nakada M, Hayashi Y (2016) Chronic spatial working memory deficit associated with the superior longitudinal fasciculus: a study using voxel-based lesion-symptom mapping and intraoperative direct stimulation in right prefrontal glioma surgery. J Neurosurg 125:1024-1032

36. Kinsbourne M (1970) A model for the mechanism of unilateral neglect of space. Trans Am Neurol Assoc 95:143-146

37. Klingberg T (2006) Development of a superior frontal-intraparietal network for visuo-spatial working memory. Neuropsychologia 44:2171-2177

38. Lawes INC, Barrick TR, Murugam V, Spierings N, Evans DR, Song M, Clark CA (2008) Atlas-based segmentation of white matter tracts of the human brain using diffusion tensor tractography and comparison with classical dissection. Neuroimage 39:62-79

39. Lemée JM, Bernard F, Ter Minassian A, Menei P (2018) Right hemisphere cognitive functions: from clinical and anatomical bases to brain mapping during awake craniotomy. Part II: neuropsychological tasks and brain mapping. World Neurosurg 118:360-367. https://doi.org/10.1016/j.wneu.2018.07.099

40. Lezak MD (1982) The problem of assessing executive functions. Int J Psychol 17:281-297

41. Lus T, Angelini E, de Schotten MT, Mandonnet E, Duffau H (2011) Evidence for potentials and limitations of brain plasticity using an atlas of functional resectability of WHO grade II gliomas: towards a "minimal common brain." Neuroimage 56:992-1000

42. MacPherson SE, Allerhand M, Cox SR, Deary IJ (2019) Individual differences in cognitive processes underlying Trail Making Test-B performance in old age: the Lothian Birth Cohort 1936. Intelligence 75:23-32

43. Maier-Hein KH, Neher PF, Houde J-C, Côté M-A, Garyfallidis E, Zhong J, Chamberland M, Yeh F-C, Lin Y-C, Ji Q (2017) The challenge of mapping the human connectome based on diffusion tractography. Nat Commun 8:1-13

44. Makris N, Kennedy DN, McInerney S, Sorensen AG, Wang R, Caviness VS Jr, Pandya DN (2005) Segmentation of subcomponents within the superior longitudinal fascicle in humans: a quantitative, in vivo, DT-MRI study. Cereb Cortex 15:854-869

45. Mandonnet E, Cerliani L, Siuda-Krzywicka K, Poisson I, Zhi N, Volle E, Thiebaut De Schotten M (2017) A network-level approach of cognitive flexibility impairment after surgery of a right temporo-parietal glioma. Neurochirurgie 63:308-313. https://doi.org/10.1016/j.neuchi.2017.03.003

46. Mandonnet E, Herbet G, Duffau H (2020) Letter: Introducing new tasks for intraoperative mapping in awake glioma surgery: clearing the line between patient care and scientific research. Neurosurgery 86:E256-E257. https://doi.org/10.1093/neuros/ nyz447

47. Mandonnet E, Sarubbo S, Petit L (2018) The nomenclature of human white matter association pathways: proposal for a systematic taxonomic anatomical classification. Front Neuroanat 12:94

48. Mandonnet E, Vincent M, Valero-Cabré A, Facque V, Barberis M, Bonnetblanc F, Rheault F, Volle E, Descoteaux M, Margulies DS (2020) Network-level causal analysis of set-shifting during trail making test part B: a multimodal analysis of a glioma surgery case. Cortex 132:238-249. https://doi.org/10.1016/j.cortex.2020. 08.021

49. Mandonnet E, Wager M, Almairac F, Baron MH, Blonski M, Freyschlag CF, Barone F, Fontaine D, Pallud J, Hegi M, Viegas C, Zetterling M, Spena G, Goodden J, Rutten GJ, Taillandier L, Foroglu N, Darlix A, Skrap M, Martino J, von Campe G, Madadaki C, Gayat E, de Witt HP, Gil Robles S, Sarubbo S, Santarius T, Bello L, Forster MT, Duffau H (2017) Survey on current practice within the European Low-Grade Glioma Network: where do we stand and what is the next step? Neurooncol Pract 4:241-247. https://doi. org/10.1093/nop/npw031

50. Mandonnet E, Winkler PA, Duffau H (2010) Direct electrical stimulation as an input gate into brain functional networks: principles, advantages and limitations. Acta Neurochir 152:185-193

51. Martino J, Gomez E, de Lucas EM, Mato D, Vázquez-Bourgon J (2018) Intraoperative identification and preservation of verbal memory in diffuse gliomas: a matched-pair cohort study. Neurosurgery 83:1209-1218

52. Mesulam MM (1981) A cortical network for directed attention and unilateral neglect. Ann Neurol 10:309-325

53. Miyake A, Friedman NP (2012) The nature and organization of individual differences in executive functions: four general conclusions. Curr Dir Psychol Sci 21:8-14. https://doi.org/10.1177/ 0963721411429458

54. Miyake A, Friedman NP, Emerson MJ, Witzki AH, Howerter A, Wager TD (2000) The unity and diversity of executive functions and their contributions to complex "frontal lobe" tasks: a latent variable analysis. Cogn Psychol 41:49-100

55. Moffitt TE, Arseneault L, Belsky D, Dickson N, Hancox RJ, Harrington $\mathrm{H}$, Houts R, Poulton R, Roberts BW, Ross S, Sears MR, Thomson WM, Caspi A (2011) A gradient of childhood self-control predicts health, wealth, and public safety. Proc Natl Acad Sci U S A 108:2693-2698. https://doi.org/10.1073/pnas.1010076108

56. Moher D, Liberati A, Tetzlaff J, Altman DG, Group P (2009) Preferred reporting items for systematic reviews and meta-analyses: the PRISMA statement. PLoS med 6 7:e1000097

57. Moore MJ, Gillebert C, Demeyere N (2021) Right-and left-lateralised neglect are not anatomically homologous: a voxel-lesion symptom mapping study

58. Motomura K, Chalise L, Ohka F, Aoki K, Tanahashi K, Hirano M, Nishikawa T, Wakabayashi T, Natsume A (2018) Supratotal resection of diffuse frontal lower grade gliomas with awake 
brain mapping, preserving motor, language, and neurocognitive functions. World Neurosurg 119:30-39. https://doi.org/10.1016/j. wneu.2018.07.193

59. Motomura K, Chalise L, Ohka F, Aoki K, Tanahashi K, Hirano M, Nishikawa T, Yamaguchi J, Shimizu H, Wakabayashi T, Natsume A (2020) Neurocognitive and functional outcomes in patients with diffuse frontal lower-grade gliomas undergoing intraoperative awake brain mapping. J Neurosurg 132:1683-1691. https://doi. org/10.3171/2019.3.JNS19211

60. Nakajima R, Kinoshita M, Okita H, Yahata T, Nakada M (2019) Glioma surgery under awake condition can lead to good independence and functional outcome excluding deep sensation and visuospatial cognition. Neuro-Oncology Practice 6:354-363. https:// doi.org/10.1093/nop/npy054

61. Ng JCH, See AAQ, Ang TY, Tan LYR, Ang BT, King NKK (2019) Effects of surgery on neurocognitive function in patients with glioma: a meta-analysis of immediate post-operative and long-term follow-up neurocognitive outcomes. J Neurooncol 141:167-182

62. Nimsky C, Bauer M, Carl B (2016) Merits and limits of tractography techniques for the uninitiated. In: Advances and technical standards in neurosurgery. Springer, pp 37-60

63. Papagno C, Comi A, Riva M, Bizzi A, Vernice M, Casarotti A, Fava E, Bello L (2017) Mapping the brain network of the phonological loop. Hum Brain Mapp 38:3011-3024. https://doi.org/10.1002/hbm. 23569

64. Perry ME, McDonald CR, Hagler DJ Jr, Gharapetian L, Kuperman JM, Koyama AK, Dale AM, McEvoy LK (2009) White matter tracts associated with set-shifting in healthy aging. Neuropsychologia 47:2835-2842

65. Posner M, DiGirolamo G (1998) Executive attention: conflict, target detection, and cognitive control. The Attentive Brain, ed R Parasuraman, Cambridge, MA: MIT Press 18:401-423

66. Puglisi G, Howells H, Sciortino T, Leonetti A, Rossi M, Conti Nibali M, Gabriel Gay L, Fornia L, Bellacicca A, Vigano L (2019) Frontal pathways in cognitive control: direct evidence from intraoperative stimulation and diffusion tractography. Brain 142:2451-2465

67. Puglisi G, Sciortino T, Rossi M, Leonetti A, Fornia L, Nibali MC, Casarotti A, Pessina F, Riva M, Cerri G, Bello L (2019) Preserving executive functions in nondominant frontal lobe glioma surgery: an intraoperative tool. J Neurosurg 131:474-480. https://doi.org/10. 3171/2018.4.JNS18393

68. Reitan RM, Wolfson D (1985) The Halstead-Reitan neuropsychological test battery: Theory and clinical interpretation. Reitan Neuropsychology 4

69. Rijnen SJ, Kaya G, Gehring K, Verheul JB, Wallis OC, Sitskoorn MM, Rutten G-JM (2019) Cognitive functioning in patients with low-grade glioma: effects of hemispheric tumor location and surgical procedure. J Neurosurg 1:1-12

70. Rolland A, Herbet G, Duffau H (2018) Awake surgery for gliomas within the right inferior parietal lobule: new insights into the functional connectivity gained from stimulation mapping and surgical implications. World Neurosurgery 112:E393-E406. https://doi.org/ 10.1016/j.wneu.2018.01.053

71. Roux FE, Dufor O, Lauwers-Cances V, Boukhatem L, Brauge D, Draper L, Lotterie JA, Démonet JF (2011) Electrostimulation mapping of spatial neglect. Neurosurgery 69:1218-1231. https://doi.org/ 10.1227/NEU.0b013e31822aefd2

72. Rutten G, Kristo G, Pigmans W, Peluso J, Verheul H (2014) The use of MR tractography in daily neurosurgical practice [article in Dutch]. Dutch J Neurol Neurosurg 115:204-211

73. Rutten G-J, Ramsey NF (2010) The role of functional magnetic resonance imaging in brain surgery. Neurosurg Focus 28:E4

74. Sarubbo S, De Benedictis A, Merler S, Mandonnet E, Balbi S, Granieri E, Duffau H (2015) Towards a functional atlas of human white matter. Hum Brain Mapp 36:3117-3136. https://doi.org/10. $1002 / \mathrm{hbm} .22832$
75. Sarubbo S, Tate M, De Benedictis A, Merler S, Moritz-Gasser S, Herbet G, Duffau H (2020) - Mapping critical cortical hubs and white matter pathways by direct electrical stimulation: an original functional atlas of the human brain. Neuroimage 205:116237

76. Schouwenaars IT, de Dreu MJ, Rutten G-JM, Ramsey NF, Jansma JM (2020) A functional MRI study of presurgical cognitive deficits in glioma patients. Neuro-Oncology Practice 8, 1:81-90

77. Sharp DJ, Beckmann CF, Greenwood R, Kinnunen KM, Bonnelle V, De Boissezon X, Powell JH, Counsell SJ, Patel MC, Leech R (2011) Default mode network functional and structural connectivity after traumatic brain injury. Brain 134:2233-2247

78. Skrap M, Marin D, Ius T, Fabbro F, Tomasino B (2016) Brain mapping: a novel intraoperative neuropsychological approach. J Neurosurg 125:877-887

79. Slim K, Nini E, Forestier D, Kwiatkowski F, Panis Y, Chipponi J (2003) Methodological index for non-randomized studies (MINORS): development and validation of a new instrument. ANZ J Surg 73:712-716

80. Smith EE, Jonides J (1999) Storage and executive processes in the frontal lobes. Science 283:1657-1661

81. Striemer C, Ferber S, Danckert J (2013) Spatial working memory deficits represent a core challenge for rehabilitating neglect. Front Hum Neurosci 7:334

82. Stroop JR (1935) Studies of interference in serial verbal reactions. J Exp Psychol 18:643

83. Stuss DT, Alexander MP (2007) Is there a dysexecutive syndrome? Philos Trans R Soc B Biol Sci 362:901-915

84. Szelényi A, Bello L, Duffau H, Fava E, Feigl GC, Galanda M, Neuloh G, Signorelli F, Sala F (2010) Intraoperative electrical stimulation in awake craniotomy: methodological aspects of current practice. Neurosurg Focus 28:E7. https://doi.org/10.3171/2009.12. FOCUS09237

85. Theeuwes $\mathbf{J}$ (2010) Top-down and bottom-up control of visual selection. Acta Physiol (Oxf) 135:77-99

86. Thiebaut de Schotten M, Urbanski M, Duffau H, Volle E, Lévy R, Dubois B, Bartolomeo P (2005) Direct evidence for a parietalfrontal pathway subserving spatial awareness in humans. Science 309:2226-2228. https://doi.org/10.1126/science.1116251

87. Tsujimoto K, Mizuno K, Nishida D, Tahara M, Yamada E, Shindo S, Kasuga S, Liu M (2019) Prism adaptation changes resting-state functional connectivity in the dorsal stream of visual attention networks in healthy adults: a fMRI study. Cortex 119:594-605

88. Valko PO, Siddique A, Linsenmeier C, Zaugg K, Held U, Hofer S (2015) Prevalence and predictors of fatigue in glioblastoma: a prospective study. Neuro Oncol 17:274-281

89. Vallar G, Bello L, Bricolo E, Castellano A, Casarotti A, Falini A, Riva M, Fava E, Papagno C (2014) Cerebral correlates of visuospatial neglect: a direct cerebral stimulation study. Hum Brain Mapp 35:1334-1350. https://doi.org/10.1002/hbm.22257

90. Van der Stoep N, Van der Stigchel S, Van Engelen RC, Biesbroek JM, Nijboer TC (2019) Impairments in multisensory integration after stroke. J Cogn Neurosci 31:885-899

91. Vanderweyen DC, Theaud G, Sidhu J, Rheault F, Sarubbo S, Descoteaux M, Fortin D (2020) The role of diffusion tractography in refining glial tumor resection. Brain Struct Funct 225:1413-1436. https:// doi.org/10.1007/s00429-020-02056-Z

92. Vandierendonck A, Liefooghe B, Verbruggen F (2010) Task switching: interplay of reconfiguration and interference control. Psychol Bull 136:601

93. Varjacic A, Mantini D, Demeyere N, Gillebert CR (2018) Neural signatures of Trail Making Test performance: evidence from lesionmapping and neuroimaging studies. Neuropsychologia 115:78-87. https://doi.org/10.1016/j.neuropsychologia.2018.03.031

94. Vavassori L, Sarubbo S, Petit L (2021) Hodology of the superior longitudinal system of the human brain: a historical perspective, the current controversies, and a proposal. Brain Struct Funct:1-22 
95. Wager M, Du Boisgueheneuc F, Pluchon C, Bouyer C, Stal V, Bataille B, Guillevin MC, Gil R (2013) Intraoperative monitoring of an aspect of executive functions: administration of the Stroop test in 9 adult patients during awake surgery for resection of frontal glioma. Oper Neurosurg 72:ons169-ons 181

96. Wechsler D (1987) Wechsler memory scale-revised: manual. The Psychological Corporation \& Harcourt Brace Jovanovich. Inc, San Antonio
Publisher's note Springer Nature remains neutral with regard to jurisdictional claims in published maps and institutional affiliations. 